\title{
Germanica
}

GERMANICA $33 \mid 2003$

Mémoire et progrès dans la littérature et l'histoire des idées allemandes au début du XXe siècle

\section{Oublier Apollon...}

Apollo vergessen

Florence Bancaud

\section{CpenEdition}

Journals

Édition électronique

URL : http://journals.openedition.org/germanica/1821

DOI : 10.4000/germanica. 1821

ISSN : 2107-0784

Éditeur

Université de Lille

Édition imprimée

Date de publication : 31 décembre 2003

Pagination : 13-35

ISBN : 9782913857124

ISSN : 0984-2632

Référence électronique

Florence Bancaud, « Oublier Apollon... », Germanica [En ligne], 33 | 2003, mis en ligne le 30 novembre 2012, consulté le 06 octobre 2020. URL : http://journals.openedition.org/germanica/1821 ; DOI : https://doi.org/10.4000/germanica.1821

Ce document a été généré automatiquement le 6 octobre 2020.

(c) Tous droits réservés 


\title{
Oublier Apollon...
}

\author{
Apollo vergessen
}

\section{Florence Bancaud}

1 Doit-on savoir oublier pour devenir créateur de sa propre existence? Telle est la réponse que Nietzsche oppose à la «maladie de l'historicisme » d'un Burckhardt ou d'un Hartmann qu'il dénonce en 1873 dans la seconde Considération intempestive où il en appelle à l'oubli salvateur et aux forces vives de la jeunesse pour régénérer la culture malade et pour retrouver le sens de l'humain :

Le plus petit comme le plus grand bonheur sont toujours créés par une chose : le pouvoir d'oublier [...]. Celui qui ne sait pas se reposer sur le seuil du moment, oubliant tout le passé [...], ne saura jamais ce qu'est le bonheur, et, ce qui pis est, il ne fera jamais rien qui puisse rendre heureux les autres ${ }^{1}$.

2 S'il est impossible de renoncer à la mémoire, l'oubli est de fait nécessaire à la vie car il peut garantir l'insouciance que l'on envie à l'animal ou à l'enfant et qui permet d'aller de l'avant :

Il est donc possible de vivre sans presque se souvenir, de vivre même heureux, à l'exemple de l'animal, mais il est absolument impossible de vivre sans oublier².

3 C'est la force plastique qui opère le tri nécessaire entre ce qu'il faut conserver, oublier, digérer ou transformer du passé pour progresser et développer une culture propre :

Pour pouvoir déterminer [...] les limites où le passé doit être oublié sous peine de devenir le fossoyeur du présent, il faudrait connaître exactement la force plastique d'un homme, d'un peuple, d'une civilisation, je veux dire cette force qui permet de se développer hors de soi-même, d'une façon qui vous est propre, de transformer et d'incorporer les choses du passé, de guérir et de cicatriser des blessures, de remplaçer ce qui est perdu, de refaire par soi-même des formes brisées ${ }^{3}$.

C'est donc en sachant prendre suffisamment de recul par rapport au passé que l'on peut tirer des leçons de l'histoire et conserver du passé ce qui, seul, peut nourrir l'avenir ; pour Nietzsche, c'est même ce pouvoir qui fait l'homme :

C'est en ayant la force d'utiliser le passé en vue de la vie et de transformer les

événements en histoire que l'homme devient homme ${ }^{4}$. 
On sait combien Nietzsche a inspiré la génération expressionniste. Nous nous proposons donc de montrer ici, en transposant cet éloge de l'oubli historique au domaine de l'esthétique, comment l'oubli volontaire du beau au profit de l'exigence du laid provoque en Allemagne au début $\mathrm{du} \mathrm{xx}^{\mathrm{e}}$ siècle une remise en cause des canons esthétiques traditionnels qui culmine dans la littérature et l'art expressionnistes et ouvre à l'artiste le champ du dionysiaque, du cri et du choc nécessaires à l'évolution de l'art vers une esthétique de l'expression et de la rébellion contre la laideur du monde.

Dans la Naissance de la tragédie, Nietzsche montre comment l'histoire de l'art est dominée par la lutte et la complémentarité entre les principes apollinien et dionysiaque. Si Apollon est le dieu de la lumière, de l'harmonie, de la belle apparence et du rêve, Dionysos est le dieu du chaos, de l'ivresse et de l'extase; il rompt le principe individuel apollinien dans les orgies dionysiaques où la nature et l'instinct triomphent. Toute l'histoire de l'art résulte d'une lutte constante entre ces deux principes :

C'est dans la lutte qui oppose les principes contraires de l'ivresse destructrice et du rêve créateur qu'apparaît le moment historique de l'art. C'est par son propre mouvement qu'il ne cesse de susciter la destruction de ce qui a été atteint, puis, aussitôt après, sa recréation à un niveau supérieur ${ }^{5}$.

7 Car c'est précisément en sachant détruire l'état précédent que l'on peut atteindre une étape supérieure: l'art comme la vie sont une création constante de la volonté de puissance, génie de la métamorphose et de la création de soi. Dans l'Essai d'autocritique qui ouvre en 1886 l'édition de la Naissance de la Tragédie, Nietzsche associe l'apollinien au beau et le dionysiaque au laid en montrant qu'au désir antique du beau et de l'harmonieux répond aussi un besoin de laideur plus ancien encore :

D'où viendrait alors la tendance contraire et chronologiquement antérieure, le désir de laideur, la sincère et âpre volonté de pessimisme des premiers Hellènes, leur volonté de mythe tragique, de mettre en image tout ce qu'il y a de terreur, de cruauté, de mystère, de destruction, de fatalité au fond de l'existence, - d'où viendrait alors la tragédie ? ${ }^{6}$

8 La tragédie répond à cette fascination pour la laideur dionysiaque et les forces du chaos et de la terre en associant au son apollinien de la cithare les dissonances dionysiaques de la flûte, destinées à susciter la terreur et la pitié du spectateur. Mais la laideur se fond ici dans le sublime tragique et participe d'une esthétique de la pitié, centrée sur l'effet, la Wirkung.

9 En revanche, dans l'œuvre tardive de Nietzsche, la laideur n'est plus une valeur affective, mais le symptôme d'une décadence de la culture occidentale dominée par le principe apollinien, où il intègre Wagner, Hugo, Baudelaire et Delacroix. Tous illustrent une esthétique décadente car nihiliste, négatrice des valeurs et affaiblissant l'homme en niant la vie :

L'une (l'esthétique classique) communique sa plénitude aux choses. Elle transfigure, elle embellit, elle rationalise le monde, l'autre appauvrit, apâlit, enlaidit la valeur des choses, elle nie le monde ${ }^{7}$.

10 La laideur est ici le signe de l'épuisement et de dégénérescence de l'homme; elle est donc pathétique et haïssable précisément parce qu'elle est le signe de l'affaiblissement de l'homme et résulte de la morale du ressentiment et de la haine de soi inhérente aux valeurs chrétiennes :

Rien n'est laid si ce n'est l'homme qui dégénère [...]. Du point de vue physiologique, tout ce qui est laid affaiblit et attriste l'homme. Cela le fait songer à la décomposition, au danger, à l'impuissance. Il y perd décidément de la force [...]. Ici, 
une haine jaillit: qui l'homme hait-il ici? Mais il n'y a à cela aucun doute : l'abaissement de son type ${ }^{8}$.

11 Ce type de laideur doit être surmonté par le beau apollinien qui permet d'enjoliver la vie et d'oublier tout ce qui affaiblit l'homme :

Tout art tonifie [...]. Le laid, c'est le contraire de l'art, ce qui en est exclu, son «non». Chaque fois que le déclin, l'appauvrissement de la vie, l'impuissance, la décomposition, la putréfaction le menacent, fût-ce de loin, l'homme esthétique y réagit en y opposant son "non ». Le laid a un effet déprimant, c'est l'expression d'une dépression. Il ôte de la force, appauvrit, opprime. [...] Le laid boîte, le laid trébuche9.

12 Mais, par un ultime renversement, Nietzsche en vient à justifier la laideur comme stimulant à la création artistique. Certes, la laideur est le symptôme essentiel du déclin des valeurs du monde moderne; mais elle peut et doit inspirer l'artiste comme le surhomme et l'inciter à rejeter ce monde et à substituer au chaos et à l'absurdité un nouveau sens, toute création supposant une destruction salvatrice de ce qui nuit à la vie et à l'homme :

J'ai moi-même cherché une justification esthétique : comment la laideur du monde est-elle possible? J'ai vu dans la volonté de beauté, la volonté de rester entouré de formes semblables un soutien et un remède provisoires: mais ce qui m'a paru fondamental, c'est l'éternel pouvoir de créer, en tant qu'éternel devoir de détruire lié à la souffrance. Le laid est la manière de contempler les choses subsumée à la volonté de donner un sens, un sens nouveau à ce qui est devenu absurde : c'est la force accumulée qui contraint le créateur à considérer le passé comme intenable et comme raté, comme objet d'une nécessaire négation, comme laid ${ }^{10}$.

13 Avec Nietzsche, Hugo et Baudelaire constituent des références essentielles pour ceux qui revendiquent cette esthétique de la modernité qui, sur fond d'angoisse et de crise, multiplie les images de la laideur et de la catastrophe pour montrer un homme en crise dans un monde décentré, qui ne croit plus en Dieu, en l'homme, en la nature ou en l'utopie et où la notion même de souveraineté du sujet devient illusoire, l'homme étant réifié dans un monde où règne en maître la rationalité technicienne. Dans la Préface de Cromwell, Hugo fait l'apologie de la laideur polymorphe contre la beauté monotone, déclarant que «le beau n'a qu'un type » et que le "laid en a mille ». Il voit dans le grotesque l'expression spécifique de la modernité, dont le canon esthétique n'est plus le beau idéal, mais la vérité :

La muse moderne verra les choses d'un coup d'oeil plus haut et plus large. Elle sentira que tout dans la création n'est pas humainement beau, que le laid y existe à côté du beau, le difforme près du gracieux, le grotesque au revers du sublime, le mal avec le bien, l'ombre avec la lumière. (La poésie) se mettra à faire comme la nature, à mêler dans ses créations, sans pourtant les confondre, l'ombre à la lumière, le grotesque au sublime, en d'autres termes, le corps à l'âme, la bête à l'esprit ${ }^{11}$.

Affirmant que le grotesque est « la plus riche source que la nature puisse ouvrir à l'art » ${ }^{12}$, il voit dans ce mode d'expression, qui inclut le laid, le difforme et le dissonant, le plus bel hommage que l'art puisse rendre à la nature et à la vérité :

Mettons le marteau dans les théories, les poétiques et les systèmes. Jetons bas ce vieux plâtrage qui masque la façade de l'art ! Il n'y a ni règles, ni modèles ; ou plutôt il n'y a d'autres règles que les lois générales de la nature qui planent sur l'art tout entier [...] La nature donc ! La nature et la vérité ${ }^{13}$.

Pour Hugo, la laideur n'est plus relative à la beauté, mais constitue une valeur en soi et permet d'accéder, par le choc et le fragment, à une nouvelle forme d'authenticité et d'union avec le cosmos : 
Le Grotesque doit nous débarrasser du poids pesant de la Beauté et en écarter la monotonie "de sa voix criarde ». Il reflète la dissonance qui s'ouvre entre le domaine animal et les sphères les plus élevées de l'homme. En faisant voler en éclats, en "fragments ", l'apparence concrète des choses, il montre précisément que le «Grand Tout » ne nous est accessible que sous forme fragmentaire, car le Tout n'est pas accordé aux mesures humaines ${ }^{14}$.

Dans Les Fleurs du Mal, beauté et laideur s'interpénètrent, brouillant ainsi, dans un geste provocateur, la définition traditionnelle en mêlant le comique, le beau, le laid, le sublime et le grotesque. Même ce qui est éthiquement négatif, comme la figure de Satan, peut devenir source de beauté pour Baudelaire, qui affirme dès la préface des Fleurs du Mal, vouloir «extraire la beauté du mal ». La quête de l'inconnu que mène Baudelaire pour lutter contre l'ennui ne peut donc que le mener à la laideur, dont Baudelaire tente de définir le plaisir dans son Choix de maximes consolantes sur l'amour :

Sachez tirer parti de la laideur elle-même [...]

Pour certains esprits plus curieux et plus blasés, la jouissance de la laideur provient d'un sentiment encore plus mystérieux qui est la soif de l'inconnu et le goût de l'horrible. C'est ce sentiment, dont chacun porte en soi le germe plus ou moins développé, qui précipite certains poètes dans les amphithéâtres et les cliniques, et les femmes aux exécutions publiques ${ }^{15}$.

17 Cette fascination de la laideur culmine dans le charme émanant de la «charogne infâme » dont sont décrits le "ventre plein d'exhalaisons », le "ventre putride » et «l'horrible infection» dans une imagerie visionnaire et cauchemardesque :

Les mouches bourdonnaient sur ce ventre putride,

D'où sortaient de noirs bataillons

Des larves, qui coulaient comme un épais liquide

Le long de ces vivants haillons ${ }^{16}$.

Mais la laideur, outre son aspect provocateur, a aussi une valeur éthique. Pour l'art moderne, qui se doit d'exprimer le mal-être de l'homme et de détruire ce qui l'opprime afin de pouvoir édifier ensuite un nouvel humanisme, le laid constitue une forme de déconstruction ou de «différance » qui instaure, au sens de Derrida, un écart critique par rapport au réel et active la conscience de crise de l'individu moderne. Ainsi Ursula Francke ${ }^{17}$ affirme-t-elle que la laideur - ainsi que toutes les formes de déconstruction telles la déformation, la destruction et l'amorphie - est précisément utilisée par les artistes à cause de son pouvoir répulsif, pour dénoncer l'absurdité du monde moderne.

\section{Vers une esthétique négative}

Matthieu Kessler définit l'esthétique nietzschéenne comme esthétique négative, dont la logique est de prendre plaisir à une expérience a priori déplaisante, puis de rechercher une satisfaction à l'insatisfaction: elle tire donc sa dynamique de l'opposition entre beauté et laideur et de la volonté de surmonter ce dualisme en faisant naître l'inspiration artistique de la laideur transmuée en forme esthétique :

L'esthétique négative signifie que le spectateur ou l'artiste tire sa joie de son insatisfaction. [...] (Elle) est même rendue nécessaire par le besoin d'oppositions et d'obstacles de toutes natures, indispensables à l'expression d'une volonté de puissance selon toutes ses formes et toutes ses possibilités ${ }^{18}$.

20 À cette esthétique moderne qui, enfreignant les canons traditionnels, renouvelle les pratiques perceptives et redéfinit l'expérience esthétique en y introduisant des catégories telles que la dissonance, la laideur ou la disharmonie, jusqu'alors perçues 
comme négatives, répond, au début $\mathrm{du} \mathrm{xx}^{\mathrm{e}}$ siècle, la littérature que Thomas Anz qualifie de littérature existentielle (Literatur der Existenz) et qui prétend répondre à la laideur du monde en rompant avec le principe apollinien de stylisation du réel et au rôle prédominant de la raison et l'intellect apolliniens et en dénonçant l'aliénation et la perte d'identité de l'individu moderne, écrasé par la technique et la rationalisation de la vie: "Bien loin d'enjoliver, d'harmoniser ou d'anticiper un monde positif et harmonieux, la littérature de l'aliénation et l'angoisse suscite un choc chez le lecteur ${ }^{19}$.»

21 La belle apparence étant désormais dénoncée comme duperie - «La littérature existentielle [...] comprend la beauté comme enjolivement, l'harmonie comme harmonisation, l'idylle bucolique comme escapisme $»^{20}$ - le changement de fonction entraîne un changement de style et l'intégration de la laideur comme sujet de l'art :

Une de ses composantes essentielles est la représentation non-esthétique du laid et du disharmonieux : la société dénonce comme laid ce qu'elle hait ou veut cacher. La représentation de la laideur obéit à la volonté de faire accéder à la conscience ce qui est publiquement refoulé ou méconnu ou, au contraire, à rompre, par la critique, avec l'illusion de ce qui est esthétisé par la culture. Sont considérées comme laides l'expression de la souffrance, la maladie du corps et de l'esprit, les grimaces de la peur ${ }^{21}$.

Lydie Krestovsky distingue ainsi dans l'art moderne trois nouvelles idées-forces: la déformation, la désintégration, la déshumanisation. La déformation, par laquelle l'artiste moderne, à partir d'un nouveau traitement de la forme, exprime la vision d'un monde qui ne peut plus être harmonieux :

La déformation sert d'explosif qui brise le modelage des formes belles et gracieuses. L'harmonie cède la place aux contours tourmentés. L'unité s'efface pour permettre l'éclosion d'un monde de visions paradoxales, d'un multipolarisme qui éblouit ${ }^{22}$.

La seconde idée-force est la désagrégation ou la fragmentation de la forme, écho au tragique moderne :

L'artiste fractionne la face et le corps de l'homme, étage des superstructures en cubes et en carrés, désagrège les objets avec le désir manifeste d'étirer, d'exagérer et de déformer les proportions des modèles quels qu'ils soient. Cette mutilation de la forme crée un monde plastique de par son essence tragique ${ }^{23}$.

24 Enfin, le troisième pivot de l'esthétique moderne est la déshumanisation, qui, défigurant l'homme, s'oppose à l'anthropocentrisme traditionnel et témoigne de la crise des valeurs et du sujet :

Le principe du rejet de l'humain dans l'art est lourd de conséquences. Il suppose l'existence d'un nouveau concept de la vie, régi par la mécanique, plié aux lois d'une abstraction. L'homme est non seulement diminué et détrôné par cette nouvelle esthétique, mais encore réduit à la caricature, au grotesque qui tuent la beauté du corps, de la face, de Dieu ${ }^{24}$.

La laideur est donc devenue le fondement de l'esthétique moderne :

Au goût des belles choses figurées par les belles formes s'est substitué le souci d'exprimer les choses telles que l'homme les voit et les sent tragiquement, telles qu'il les conteste ou les dénonce dramatiquement pour les traduire en des formes tourmentées ${ }^{25}$.

Dans Les Cahiers de Malte Laurids Brigge, Rilke fait ainsi de l'écriture la tentative de dépasser les apparences dénoncées comme un décor vain et grotesque et de pénétrer dans l'essence intime des choses : 
Est-il possible qu'en dépit de toutes les inventions et de tous les progrès, qu'en dépit de la civilisation, de la religion, de la philosophie, on en soit resté à la surface de la vie ?26 critère suprême de l'art, fait l'éloge du laid comme du caractéristique :

Le vulgaire s'imagine volontiers que ce qu'il juge laid dans la réalité n'est pas matière artistique. Il voudrait nous interdire de représenter ce qui lui déplaît et l'offense dans la Nature. C'est une profonde erreur de sa part. Ce qu'on nomme communément laideur dans la nature peut dans l'art devenir d'une grande beauté. 
Dans l'ordre des choses réelles on appelle laid ce qui est difforme, ce qui est malsain, ce qui suggère l'idée de la maladie, de la débilité et de la souffrance, ce qui est contraire à la régularité, signe et condition de la santé et de la force [...] Mais qu'un grand artiste ou un grand écrivain s'empare de l'une ou de l'autre de ces laideurs, instantanément il la transfigure... d'un coup de baguette magique il en fait de la beauté : c'est de l'alchimie, de la féerie ! ${ }^{31}$

Pour Rodin en effet, « est beau uniquement ce qui a du caractère. Le caractère, c'est la vérité intense d'un spectacle naturel quelconque, beau ou laid. [...]. Et comme c'est uniquement la puissance du caractère qui fait la beauté de l'art, il arrive souvent que plus un être est laid dans la Nature, plus il est beau dans l'Art ${ }^{32}$. » Rodin inverse ainsi les canons traditionnels du beau et du laid, jugeant laid dans l'art que ce qui est sans caractère, faux et artificiel, bref, ce qui nuit à la vérité. L'art moderne se doit d'être l'expression de la vie sous toutes ses formes, fût-ce des formes disgracieuses, car ce qui importe pour Rodin est de voir et de faire voir ce qui existe, au risque de bousculer les préjugés établis et de choquer le spectateur.

Contemporain de Rilke et, comme lui, pragois de langue allemande, Franz Kafka prône lui aussi une esthétique du choc dans une lettre à Oskar Pollak :

Il me semble d'ailleurs qu'on ne devrait lire que les livres qui vous mordent et vous piquent. Si le livre que nous lisons ne nous réveille pas d'un coup de poing sur le crâne, à quoi bon le lire ? [...] Nous avons besoin de livres qui agissent sur nous comme un malheur dont nous souffririons beaucoup, comme la mort de quelqu'un que nous aimerions plus que nous-mêmes, [...] comme un suicide - un livre doit être la hache qui brise la mer gelée en nous ${ }^{33}$.

Max Brod, ami et exécuteur testamentaire de Kafka, évoque également sa fascination pour des tableaux anti-académiques admirés en 1913 à la maison des artistes (Künstlerhaus) de Vienne, se faisant l'apologue du mauvais goût et du choc salutaire parce qu'entraînant une conscience accrue du réel :

Depuis toujours, j'aime le confort, la grâce inconsciente de mauvaises images, cette ironie qui ignore tout d'elle-même, cette élégance des effets involontaires [...]. Elles sont si univoques, si parfaites, si laides... les belles images. Mais ce sont les délices d'un ballet instinctif, la nature involontaire et inépuisable, le chaos et les cérémonies primitives que je lis dans les clichés des annonces, dans les images des réclames, dans les timbres, dans les vignettes autocollantes, dans les décors des théâtres d'enfants, les décalcomanies et les vignettes: ce qui me ravit, c'est le romantisme du mauvais goût ${ }^{34}$.

35 C'est en vertu de cette exigence de choquer le lecteur et de l'arracher au confort des images lénifiantes que, dans le début de la Métamorphose, Kafka brise délibérément avec l'esthétique apollinienne du rêve et de la belle apparence en donnant à la réalité même l'apparence d'un cauchemar tout en soulignant que Grégoire Samsa est bien sorti du monde du rêve :

Un matin, au sortir d'un rêve agité, Grégoire Samsa s'éveilla transformé dans son lit en une véritable vermine. [...] « Que m'est-il arrivé ? » pensa-t-il. Ce n'était pourtant pas un rêve ${ }^{35}$.

Et c'est de ce cauchemar que part Kafka pour créer une esthétique du choc et du désenchantement allant de pair avec le désenchantement du monde (Entzauberung der Welt) dénoncé par Max Weber et l'exacerbation de la nervosité dans les villes modernes constatée par Simmel. Ce monde étant celui de la rationalité technicienne, de la bureaucratie toute puissante du Château ou du Procès ou des mégalopoles modernes tel le New York où se perd Karl Rossmann dans Le Disparu, l'homme ne peut plus y trouver de refuge dans l'amour, le rêve ou ne fût-ce que le monde harmonieux de la nature 
dénoncée comme illusion et comme obstacle à la réflexion dans l'Allocution au paysage de Description d'un Combat :

Le paysage me gêne dans mes pensées [...], il fait vaciller mes réflexions [...]. Il est beau et veut, pour cette raison, qu'on le contemple. [...] « Montagne, je ne t'aime pas, car tu me rappelles les nuages, le crépuscule et le ciel déclinant et ce sont là des choses qui me font presque pleurer car on ne peut jamais les atteindre ${ }^{36}$.

Chez les expressionnistes, contemporains de Kafka, on peut même parler d'une esthétique du cri, telle qu'elle est définie par Hermann Bahr :

Jamais l'homme n'a été aussi petit. Jamais il n'a éprouvé autant d'angoisse. Soudain, sa misère éclate dans un grand cri : dans ce cri, l'homme cherche à retrouver l'âme qu'il a perdue, le temps tout entier devient un grand cri d'angoisse. L'art lui-même joint ses cris à ceux de l'homme, dans la profondeur des ténèbres, il crie à l'aide, lance un cri d'appel à l'esprit : c'est cela, l'expressionnisme ${ }^{37}$.

Nul hasard si les peintres expressionnistes les plus représentatifs de la laideur, Meidner, Dix, Beckmann, ont tous été profondément marqués par les œuvres de Nietzsche, et notamment par le Zarathoustra. Renonçant à un art apollinien fondé sur la belle apparence et la totalité d'un monde clos et harmonieux, ils optent pour un art dionysiaque, distordant les formes, brisant la perspective, détruisant les repères visuels traditionnels afin de provoquer chez le spectateur le choc nécessaire à la destruction des schémas et canons traditionnels et à la construction d'une dynamique où la vitalité le dispute à la violence expressive et où l'éclatement des formes dénonce la laideur de la société moderne.

39 Meidner est un des premiers représentants de l'esthétique de la laideur expressionniste. Cette laideur, il l'observe d'abord sur son propre visage, en lequel il voit un masque grimaçant qu'il se plaît à mettre en scène :

Il se dégoûte lui-même, se hait lui-même, se fait des grimaces devant la glace et regarde, tel un chasseur à l'affût, sa calvitie « luisante comme un glacier ». Il se chasse lui-même. C'est avec une précision impitoyable et enragée qu'il étudie sa physionomie. Il prend plaisir à grimacer et c'est sous la contrainte de cet impitoyable plaisir de l'observation de soi qu'il commence à peindre, ricanant sur lui-même, un spectral autoportrait dont il oppose le sauvage rictus au monde entier $^{38}$ 


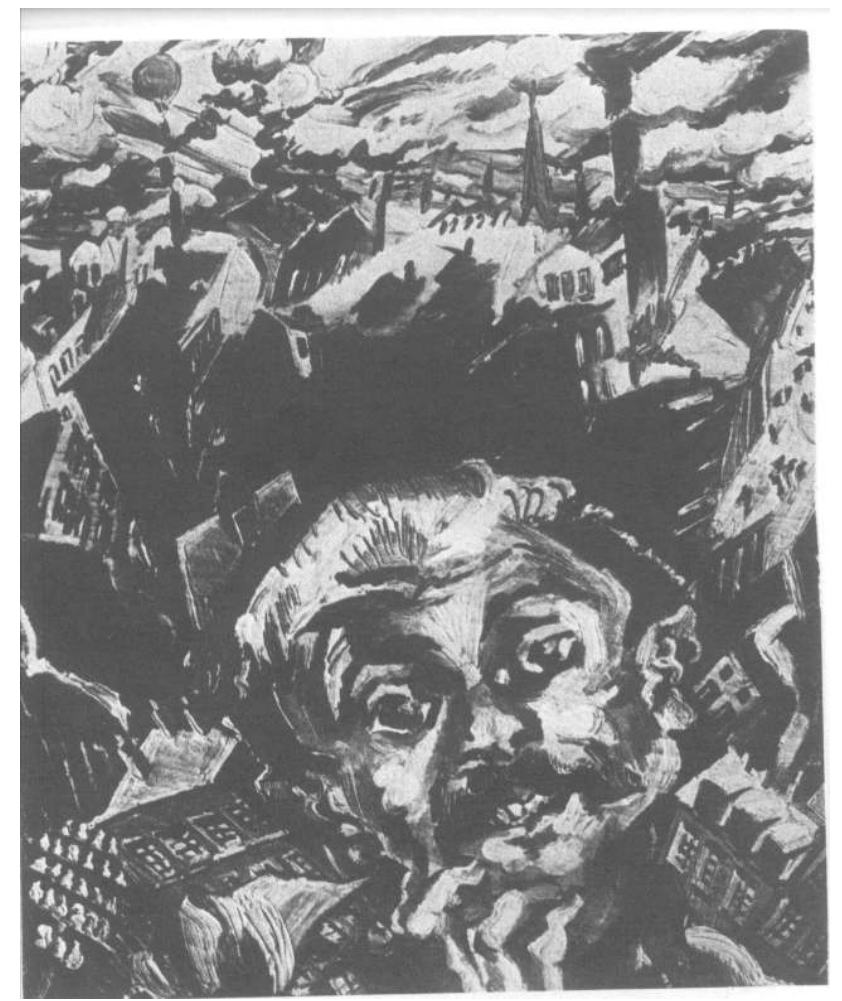

Ludwig Meidner : Ich und die Stadt (1913)

En 1912, dans son guide consacrée à la peinture des grandes villes (Anleitung zum Malen von Großstadtbildern), il fait, dans un geste provocateur, l'éloge du monde technique et industriel des métropoles où il voit le sujet d'un nouvel art, déclarant que la mission du peintre moderne est de peindre sous forme de gigantesques fresques le merveilleux, l'étrange, le monstrueux et le dramatique qui se dégagent des innombrables avenues, gares, fabriques et tours qu'il observe dans les métropoles modernes. Et, faisant écho à l'éloge de Marinetti sur la beauté de la vitesse et la supériorité esthétique d'une voiture de course sur la statue de Samothrace victorieuse, il affirme que la force dramatique d'une cheminée d'usine peinte avec expression est bien plus émouvante que bien des toiles de Raphaël. Pour saisir et représenter la dynamique des villes modernes, il invente un nouveau langage pictural recourant à des lignes de force brisées, des diagonales éclatées, des courbes interrompues et visant, dans ses tableaux apocalyptiques, à rendre compte de la fragmentation du sujet, la dispersion et l'éclatement de la perspective dans la perception moderne. Dans son autoportrait intitulé Moi et la ville (Ich und die Stadt (1913)), le visage du peintre se dresse ainsi, telle une gigantesque tête de méduse, devant des lignes de forces explosives et des diagonales brisées par l'enchevêtrement de fenêtres, d'immeubles qui s'écroulent et de cheminées d'usines fumantes: cette image apocalyptique de l'homme livré aux puissances destructrices de la ville, Walter Benjamin la saisit aussi dans son essai Expérience et pauvreté (Erfahrung und Armut), évoquant la désintégration de tous les repères de l'individu, la déshumanisation de l'homme et l'appauvrissement de l'expérience au profit du triomphe de la technique au lendemain de la Première Guerre mondiale :

Une génération qui était encore allée à l'école en tramway hippomobile se retrouvait à découvert dans un paysage où plus rien n'était reconnaissable, hormis 
les nuages et, au milieu, dans un champ de forces traversé de tensions et d'explosions destructrices, le minuscule et fragile corps humain ${ }^{39}$.

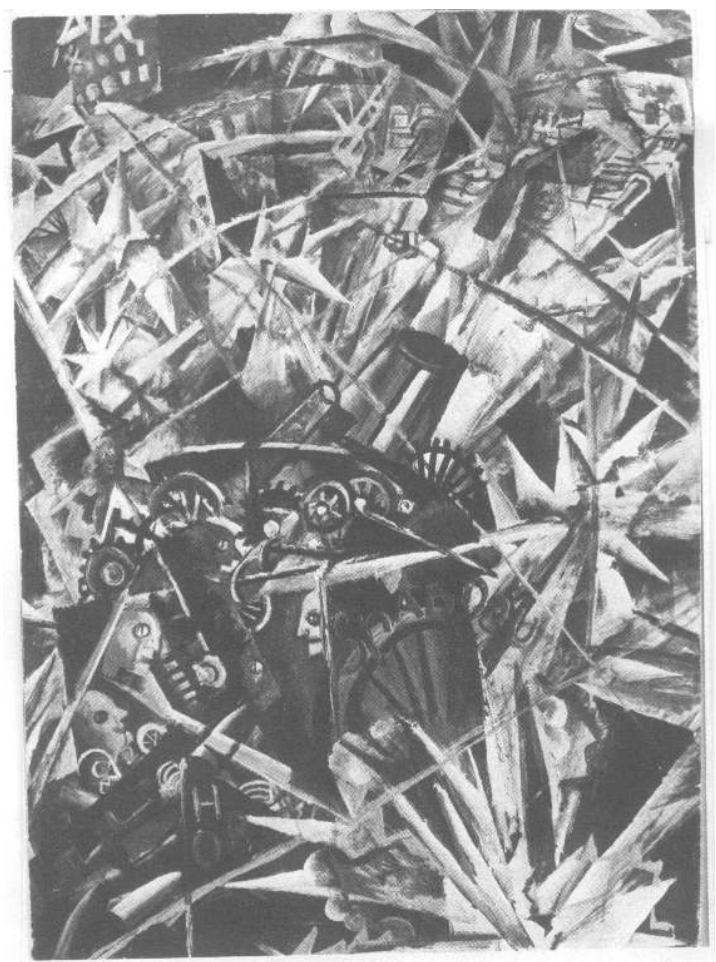

Otto Dix: Der Krieg (1919). Düsseldorf, Kunstmuseum

Otto Dix : Der Krieg (1919). Düsseldorf, Kunstmuseum

41 Si l'esthétique de la laideur de Meidner est d'abord une provocation lancée contre l'esthétique classique, le choix du laid, chez Georges Grosz et Otto Dix, résulte aussi d'une volonté éthique: ils entendent détruire le mythe de la belle apparence jusqu'alors de mise dans la culture et l'art bourgeois en démasquant la collusion entre le capitalisme, la normativité bourgeoise et l'académisme officiel: reprochant à la peinture française de n'être qu'une belle peinture, ils proclament nécessaire le retour aux sources sauvages de l'art en usant de la simplification des traits, de la distorsion des corps, de la déformation de l'image. Le propos d'Otto Dix est avant tout de démasquer les laideurs du monde. Dans La guerre (Der Krieg), une huile sur toile de 1914, l'écartèlement des formes naît de la tension explosive entre les détails qui composent l'image : il s'agit des roues d'un mécanisme absurde qui s'entrechoquent avec des armes explosives, des fragments de visages robotiques aux yeux troués, des maisons en flammes et murs effondrés, pitoyables reliques d'un monde anonyme, déshumanisé, par lesquelles Otto Dix dénonce la guerre comme destruction totale de l'humain et symptôme de la victoire de la machine sur l'homme.

Comme Otto Dix, Max Beckmann use de la déformation des formes et recourt à des couleurs crues pour représenter la réalité cruelle qui l'entoure et la vie sauvage qui bouillonne sous la croûte de la culture bourgeoise. Fortement marqué par les écrits de Nietzsche, Beckmann considère que c'est par le combat et la destruction seules que l'homme peut dépasser la morale et l'esthétique bourgeoises et renouveler la société et l'homme. C'est pourquoi il renonce à l'esthétique idéaliste de l'ornement pour montrer la réalité dans toute sa nudité et se confronter au réel pour le surmonter en le dénonçant : 
Toute forme d'apparition du monde visible apparaît comme un défi lancé à l'ordre moral. La peinture se libère des normes esthétiques pour se faire acte éthique ${ }^{40}$.

Prétendant rivaliser avec Picasso, il entend représenter les formes les plus extrêmes de la souffrance humaine et de l'horrible réalité du monde. De fait, il développe, à partir de son expérience traumatique de la guerre de 1914, un nouveau langage esthétique en se référant à des modèles gothiques du Moyen Âge tardif tels Matthias Grünewald ou Le Greco dont il admire la "mystique virile ", la difformité des personnages et la force expressive. Dans Résurrection (Auferstehung, 1916-1918), la première œuvre où il applique cette esthétique de la laideur et de l'outrance expressive, les figures nues, distordues et disproportionnées gesticulent, impuissantes et la juxtaposition de fragments heurtés témoigne de l'horreur et de la solitude extrême des personnages dont sont mis au jour la fragilité et le désespoir :

Les personnages, avec leurs tendons crispés, leurs membres excessivement étirés et leurs gestes anguleux montrent qu'ils ne sont plus les protagonistes d'un grand et noble combat pour la survie, mais les prisonniers de leurs propres contradictions intérieures. Le corps devient un fardeau, une torture dans un monde dont les repères porteurs de sens ont disparu ${ }^{41}$.

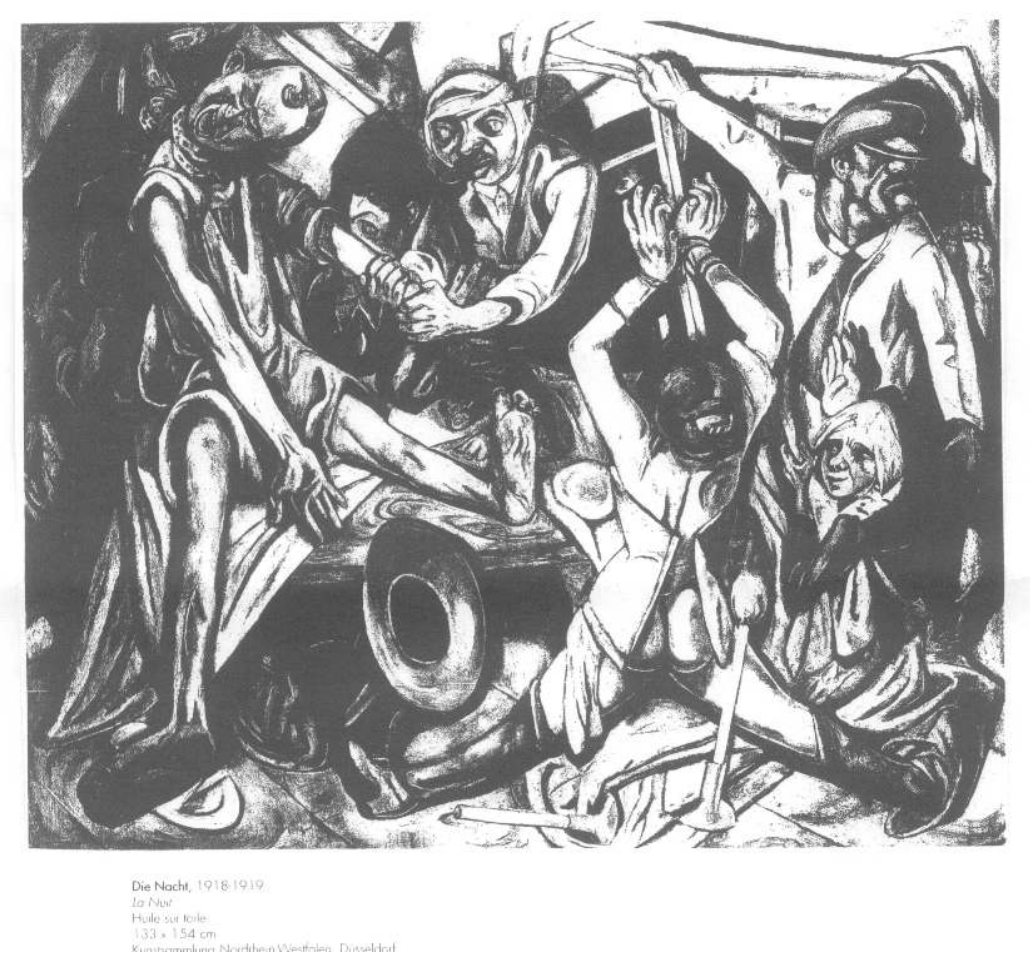

Die Nacht, 1918-1919, La Nuit.

Huile sur toile 133×154 cm. Kunstsammlung Nordrhein-Westfalen, Düsseldorf.

Le cauchemar, la torture et le meurtre hantent également La Nuit (Die Nacht, 1918-1919), où le nouveau langage visuel de Beckmann trouve son accomplissement et cristallise la révolte de l'artiste contre Dieu. Il y représente un monde chaotique et disloqué, où trois personnages s'introduisent avec une violence inouïe dans une mansarde où ils torturent un couple et enlèvent leur fille. Beckmann n'exclut ici aucun détail sordide : il n'oublie ni la mèche de cheveux sur la tête du pendu aux traits déformés par la suffocation, ni le couteau par terre, ni le chandelier renversé, ni les membres écartelés de la femme, comme dans les supplices médiévaux. Dans cette œuvre jugée par 
Siegfried Kracauer comme une allégorie de «l'instinct sanguinaire qui rôde dans les nuits et provoque des pogroms ", Beckmann réussit paradoxalement à atteindre la plus grande force d'expression de la vie dans des images dont l'atrocité cristallise la souffrance humaine et démasque l'horreur d'un monde que Beckmann a toujours perçu comme menaçant :

Je ne hais rien tant que le sentimentalisme. Plus mon désir de rapporter les choses inexprimables de la vie croît et s'intensifie, plus la détresse que m'inspire notre existence brûle en moi lourdement et profondément, [...], plus je suis envahi par le désir froid de m'emparer de cet effrayant monstre de vitalité, agité de mille soubresauts, et de l'enfermer dans la cage de mes lignes et de mes surfaces anguleuses et translucides, de le supprimer, de l'étouffer. [...] Une main peinte ou dessinée, un visage en pleurs ou grimaçant, voilà mon credo. Si j'ai senti quelque chose de la vie, c'est là̀2 .

Carl Einstein interprète La Nuit comme une image du désenchantement et de la mort des utopies :

La Nuit, c'est l'agression, le coupe-gorge, la terreur : tout autre chose que la bonne Lune avec ses elfes et les murmures des sources. Se donne à voir ce que veut le voisin : vous pendre; seulement dans son sommeil, bien entendu. Massacre avec grammophone et tabac du Palatinat; en rêve, il faut l'avouer ${ }^{43}$.

\section{La catharsis du choc}

Si l'art moderne recourt au choc esthétique et semble oublier que la vocation initiale de l'esthétique était le beau, ce n'est pas seulement par goût de la provocation et de la protestation contre la belle apparence classique. Mais c'est surtout pour amener l'homme à prendre conscience de ce qui l'opprime et pour démasquer les systèmes de pensée préétablis qui font obstacle à la prise de conscience de son aliénation. C'est ce que rappelle Thomas Anz évoquant le nouveau rôle de la « littérature existentielle » :

Ce qui suscite rejet et dégoût dans la littéraure existentielle ne relève pas [...] d'une totalisation de l'esthétique qui tend aussi à intégrer la laideur, mais est l'expression d'une nouvelle fonction de la littérature: elle devrait [...] non opposer aux disharmonies et aux contradictions un monde positif, mais en dénoncer les effets existentiels, c'est-à-dire en s'opposant à une société nécessairement attachée à cacher ses contradictions sous le masque de l'idéologie. La littérature ne devrait pas enjoliver l'existence humaine, ni la rendre plus agréable et plus supportable. C'est ce devoir dont s'était déjà, sous une forme pervertie, chargée l'industrie culturelle dominante. Mais elle devrait troubler, provoquer, détruire radicalement les systèmes de pensée et d'accomplissement établis ${ }^{44}$.

Par-delà le choc et précisément en représentant ce qui nie l'homme, la littérature comme l'art modernes se veulent ainsi non seulement une force de refus, mais aussi l'espace d'une quête de sens et de recréation d'une culture humaniste :

Sur fond de mutation dynamique de la société, la négativité de la littérature existentielle parait, dans un sens historique large, fonctionnelle, en ce que c'est dans la prise de conscience et la volonté de faire prendre conscience de la situation anachronique et désespérée des schémas de pensée existants qu'elle suscite la recherche du nouveau. La littérature existentielle est, dans la modernité, une instance qui exige de la société le questionnement permanent de ses normes, ses valeurs et ses schémas d'interprétation ${ }^{45}$.

Pour Adorno, les œuvres d'art, qui sont à la fois témoins et adversaires du déclin de l'art, doivent dénoncer l'aliénation culturelle et la fétichisation de la marchandise en 
utilisant la laideur pour résister à la pensée réconciliatrice et utilitariste de la culture bourgeoise. Le beau a donc besoin de sa négation, le laid :

Dire que l'art ne s'identifie pas au concept du beau, mais qu'il a besoin du laid, sa négation, est un lieu commun. Mais le laid, en tant que canon d'interdits, n'en disparaît pas pour autant. [...] L'interdiction du laid est devenue celle de ce qui n'est pas formé hic et nunc, de ce qui n'est pas élaboré ; elle est devenue l'interdiction de ce qui est brut. La dissonance est le terme technique qui désigne le fait que l'art intègre ce que l'esthétique, ainsi que la naïveté, appelle le laid. Quelle que soit sa nature, la laid doit constituer ou pouvoir constituer un moment de l'art ${ }^{46}$.

Adorno, pour qui le laid comme le beau constituent un moment nécessaire de l'art, associe d'abord la laideur au prétendu "progrès » incarné par le monde capitaliste, mais qui se solde en fait par la suprématie de la technique, par l'écrasement de l'homme par la machine et par un paysage ravagé par l'industrie :

Même la condamnation naïvement portée par la conscience bourgeoise à l'égard de la laideur du paysage bouleversé par l'industrie établit une relation, la domination visible de la nature, là où celle-ci présente aux hommes la façade du non-dominé. Une telle laideur disparaîtrait si le rapport des hommes à la nature se débarrassait un jour de son caractère répressif qui perpétue l'oppression de l'homme et non l'inverse. Dans un monde ravagé par la technique, cette disparition de la laideur est potentiellement contenue dans une technique pacifiée et non dans des enclaves planifiées ${ }^{47}$.

Mais la laideur doit aussi être intégrée à l'œuvre d'art pour dénoncer la laideur du monde en la reproduisant :

L'art doit faire son affaire de ce qui est mis à l'index en tant que laid, non pour l'intégrer, l'atténuer ou le réconcilier avec son existence grâce à cette chose repoussante entre toutes qu'est l'humour, mais pour dénoncer dans le laid le monde qui le crée et le reproduit à son image ${ }^{48}$.

51 L'art doit donc dénoncer dans le laid la dégénérescence du monde dont il est victime, mais dont il se libère en la stigmatisant et en refusant toute conciliation avec elle. La laideur participe donc du « désenchantement du monde » stigmatisé par Max Weber. Mais si l'art court le risque d'y perdre son aura, il gagne, en tirant parti de la laideur, une force de contestation et de dissonance qui témoignent de sa liberté et de son autonomie dans un monde où harmonie et réconciliation riment souvent avec passivité et soumission. Savoir oublier la beauté de l'apparence harmonieuse pour lui préférer la laideur de la révolte et de la non-conciliation avec le principe de violence et de destruction à l'œuvre dans la technique et le paysage industriel modernes est un gage de survie de l'art et de garantie d'un regard certes désenchanté, mais néanmoins dynamique, lucide et réaliste sur le monde.

Loin de constituer une forme de régression vers l'archaïque ou d'option pour une esthétique nihiliste ou pessimiste, l'oubli nécessaire d'Apollon et d'une esthétique de la belle apparence au profit d'une esthétique de la laideur est témoignage de progrès et promesse d'avenir. Car par-delà le laid, le difforme, la choc et le cri, c'est bien l'homme qu'il s'agit de retrouver. Le renoncement au beau a été nécessaire pour parvenir à une esthétique progressiste qui rappelle à l'homme qu'il est sujet de son histoire, de sa vie et de sa création et qu'il peut par l'art dénoncer les laideurs de l'existence et les ravages du progrès technique pour mieux les surmonter et d'en faire des stimulants à la vie future : à l'injonction nietzschéenne invitant à surmonter la laideur moderne pour créer un nouvel humanisme répond ainsi le principe expressionniste si bien résumé par 
Michel Ribon : «pour rêver d'un monde nouveau, il faut d'abord mettre en scène la hideur de l'existence réelle $»^{49}$.

\section{NOTES}

1. Friedrich Nietzsche, Seconde considération intempestive, trad. P.Y. Bourdil, Paris, Flammarion, 1978, p. 77/Vom Nutzen und Nachteil der Historie für das Leben, Stuttgart, Reclam, 1970, S. 9 : « Bei dem kleinsten aber und bei dem größten Glücke ist es immer eins, wodurch Glück zum Glücke wird: das Vergessenkönnen [...]. Wer sich nicht auf der Schwelle des Augenblicks, alle Vergangenheiten vergessend, niederlassen kann [...], der wird nie wissen, was Glück ist, und noch schlimmer : er wird nie etwas tun, was andre glücklich macht. »

2. Ibid., p. 78/S. 10 : «Es ist möglich, fast ohne Erinnerung zu leben, ja glücklich zu Leben, wie das Tier zeigt ; aber es ist ganz und gar unmöglich, ohne Vergessen überhaupt zu leben ».

3. Ibid., p. 78/S. 10 : «Um [...] die Grenze zu bestimmen, an der das Vergangene gemessen werden muß, [...] müsste man genau wissen, wie groß die plastische Kraft eines Menschen, eines Volkes, einer Kultur ist ; ich meine jene Kraft, aus sich heraus eigenartig zu wachsen, Vergangenes und Fremdes umzubilden und einzuverleiben, Wunden auszuheilen, Verlorenes zu ersetzen, zerbrochene Formen aus sich nachzuformen. "

4. Ibid., p. 80-81 (trad. Revue par nous-même)/S.12 : « Erst durch die Kraft, das Vergangene zum leben zu gebrauchen und aus dem Geschehenen wieder Geschichte zu machen, wird der Mensch zum Menschen ».

5. Volker Gerhardt, Pathos und Distanz. Studien zur Philosophie Friedrich Nietzsches, Stuttgart, Reclam, 1988, S. 31 : «Im Kampf der gegensätzlichen Prinzipien von auflösendem Rausch und gestaltendem Traum kommt ein geschichtliches Moment in die Kunst. Durch ihre eigene Bewegung führt sie immer wieder zur Destruktion des einmal Erreichten mit anschließender Neuschöpfung auf höherem Niveau. »

6. F. Nietzsche, Essai d'autocritique, $\$ 4$, in : La naissance de la tragédie, in : Cuuvres, édition dirigée par Jean Lacoste et Jacques Le Rider, Paris, Robert Laffont, 21994, p. 26/Versuch einer Selbstkritik, in : Die Geburt der Tragödie aus dem Geiste der Musik, Stuttgart, Reclam, 1953, S. 7-8 : « Woher müßte dann das entgegengesetzte Verlangen, das der Zeit nach früher hervortrat, stammen, das Verlagen nach dem Hässlichen, der gute strenge Wille des älteren Hellenen zum Pessimismus, zum tragischen Mythos, zum Bilde alles Furchtbaren, Bösen, Rätselhaften, Vernichtenden, Verhängnisvollen auf dem Grunde des Daseins - woher müsste dann die Tragödie stammen?» 7. F. Nietzsche, Le Cas Wagner, épilogue, in : Euvres, trad. Henri Albert, Paris, Flammarion, 2000, p. 1014/Der Fall Wagner, Kritische Studienausgabe (KSA), Bd. 6, Hrsg. Von Giorio Colli und Mazzimo Montinari, Berlin, de Gruyter, 1988, S. 51 : «Die erstere (die klassische Ästhetik) giebt aus ihrer Fülle an die Dinge ab - sie verklärt, sie verschönt, sie vernünftigt die Welt -, die letztere verarmt, verblasst, verhässlicht den Werth der Dinge, sie verneint die Welt. »

8. F. Nietzsche, Le crépusucule des idoles, § 20, trad. H. Albert, in : Euvres, op. cit., p. 1091/Götzendämmerung, KSA 6, § 20, S. 124 : « Nichts ist hässlich als der entartende Mensch [...]. Physiologisch nachgerechnet, schwächt und betrübt alles Hässliche den Menschen. Es erinnert ihn an Verfall, Gefahr, Ohnmacht [...]. ein Hass springt da hervor: wen hasst der Mensch ? Aber es ist kein Zweifel : den Niedergang seines Typus. » 
9. F. Nietzsche, Nachgelassene Fragmente, 1887-1889, KSA 13, S. 296 : « Alle Kunst wirkt tonisch [...]. Das Hässliche, d.h. der Widerspruch der Kunst, das, was ausgeschlossen wird von der Kunst, ihr Nein. Jedes Mal, wenn der Niedergang, die Verarmung an Leben, die Ohmacht, die Auflösung, die Verwesung von fern nun angeregt wird, reagiert der ästhetische Mensch mit seinem Nein Das Hässliche wirkt depressiv, es ist der Ausdruck einer Depression. Es nimmt Kraft, es verarmt, es drückt. [...] Das Hässliche hinkt, das Hässliche stolpert »

10. F. Nietzsche, Nachgelassene Fragmente 1885-1887, KSA 12, S. 405 : «Ich selbst habe eine ästhetische Rechtfertigung versucht : wie ist die Hässlichkeit der Welt möglich ? Ich nahm den Willen zur Schönheit, zum Verharren in gleichen Formen, als ein zeitweiliges Erhaltungs- und Heilmittel : fundamental aber schien mir das Ewig-Schaffende als das ewig Zerstören-Müssende gebunden an den Schmerz. Das Hässliche ist die Betrachtungsform der Dinge unter dem Willen, einen Sinn, einen neuen Sinn in das sinnlos Gewordene zu legen : die angehäufte Kraft, welche den Schaffenden zwingt, das Bisherige als unhaltbar, missraten, verneinungswürdig, als hässlich zu fühlen. »

11. Victor Hugo, Préface de Cromwell (1827), in : Théâtre Complet, vol. I, édition établie et annotée par J.J. Thierry et Josette Mélèze, Paris, Gallimard, Bibliothèque de la Pléiade, 1985, p. 416.

12. Ibid., p. 419.

13. Ibid., p. 434-435.

14. Hugo Friedrich, op. cit., p. 41.

15. Charles Baudelaire, Choix de maximes consolantes sur l'amour (1846), in : Cuvres Complètes, Paris, Seuil, 1968, p. 263-264.

16. Baudelaire, Une charogne, XXIX, in : Les Fleurs du Mal, Spleen et idéal, op. cit., p. 57-58.

17. Ursula Francke, "Das Hässliche», in: Historisches Wörterbuch der Philosophie, Hrsg. Von Joachim Ritter, Basel, Stuttgart, 1974, S. 1006.

18. M. Kessler, L'Esthétique de Nietzsche, Paris, PUF, 1998, p. 23.

19. Thomas Anz, Literatur der Existenz, Literarische Psychopathographie und ihre soziale Bedeutung im Frühexpressionismus, Stuttgart, Metzler, 1977, S. 167 : «Anders als verschönernd, ausgleichend oder eine positive, harmonische Welt antizipierend, wirkt die Literatur der Entfremdung und Angst anstoßerregend. »

20. Ibid., p. 169 : «Die Existenzliteratur [...] wertet Schönheit als Beschönigung, Harmonie als Harmonisierung, das (ländliche) Idyll als Realitätsflucht ».

21. Ibid., p. 169 : « Zu seinen charakteristischen Merkmalen gehört die unästhetische Darstellung des Häßlichen und Disharmonischen : als häßlich denunziert die Gesellschaft, was sie hasst oder verbergen will. Darstellung des Häßlichen mit Bewusstmachung des öffentlich Verdrängten und verkannten oder aber kritische Desillusionierung des von der Kultur Ästhetisierten. Als hässlich gelten der Ausdruck des Leidens, die Krankheit des Körpers und des Geistes, die Grimassen der Angst. "

22. L. Kreskovsky, op. cit., p. 12.

23. Ibid., p. 12.

24. Ibid., p. 13.

25. M. Ribon, op. cit., p. 16.

26. Rainer Maria Rilke, Les Carnets de Malte Laurids Brigge/trad. Claude David, in : Récits en prose, Paris, Gallimard (Bibl. de la Pléiade), 1993, p.448/Die Aufzeichnungen des Malte Laurids Brigge, Frankfurt/Main, Insel, 1995, s. 24 : « Ist es möglich, daß man trotz Erfindungen und Fortschritten, trotz Kultur, Religion und Weltweisheit an der Oberfläche des Lebens geblieben ist?»

27. Ibid., p. 464/S. 47 : «Da standen die Muttage und die Krankheiten und das Ausgeatmete und der jahrealte Rauch und der Schweiß [...]. da stand das Scharfe vom Urin und das Brennen von Ruß und grauer Kartoffeldunst [...]. Der süße, lange Geruch von vernachlässigten Säuglingen war da und der Angstgeruch der Kinder. » 
28. Ibid., p. 435-436/S. 7 : «Ich habe einen Menschen gesehen, welcher schwankte und umsank. [...] Ein Kind in einem stehenden Kinderwagen : es war dick, grünlich und hatte einen deutlichen Ausschlag auf der Stirn. [...] Das Kind schlief, der Mund war offen, atmete Jodoform, Pommes frites, Angst. »

29. Ibid., p. 459/S. 40 : «Es sind Abfälle, Schalen von Menschen, die das Schicksal ausgespieen hat. "

30. Ibid., p. 481/S. 71 : « Erinnerst du dich an Baudelaires unglaubliches Gedicht Une Charogne? Es kann sein, daß ich es jetzt verstehe. [...] Es war seine Aufgabe, in diesem Schrecklichen, scheinbar widerwärtigen, das Seiende zu sehen, das unter allem Seienden gilt. Auswahl und Ablehnung gibt es nicht. Hältst Du es für einen Zufall, daß Flaubert seinen Saint-Julien-l'Hospitalier geschrieben hat? Es kommt mir vor, als wäre das das Entscheidende : ob einer es über sich bringt, sich zu dem Aussätzigen zu legen und ihn zu erwärmen mit der Herzwärme der Liebesnächte. »

31. Rodin, L'Art, Chapitre II : " Pour l'artiste, tout est beau dans la nature », Paris, Grasset (Cahiers Rouges), 1911, p. 37.

32. Ibid., p. 39-40.

33. Kafka, Lettre à Oskar Pollak, 27 janvier 1904, in : Cuvres III, trad. M. Robert, C. David, Paris, Gallimard (Bibliothèque de la Pléiade), p.575/Brief, 27. Januar 1904, in: Briefe 1902-1924, Frankfurt, Fischer, 1983, S. 27-28 : «Ich glaube, man sollte überhaupt nur solche Bücher lesen, die einen beißen und stechen. Wenn das Buch, das wir lesen, uns nicht mit einem Faustschlag auf den Schädel weckt, wozu lesen wir dann das Buch ? [...] Wir brauchen aber die Bücher, die auf uns wirken wie ein Unglück, das uns sehr schwach, wie der Tod eines, den wir lieber hatten als und [...] wie ein Selbstmord, ein Buch muß die Axt sein für das gefrorene Meer in uns. »

34. Max Brod, Über die Schönheit hässlicher Bilder. Ein Vademecum für Romantiker unserer Zeit, Wien, Hamburg, Paul Zsolnay Verlag, 1967, S.10: «Seit damals liebe ich die Behaglichkeit, die unbewusste Grazie schlechter Bilder, diese Ironie, die von sich selbst nichts weiß, diese Eleganz der unbeabsichtigten Effekte. [...] Sie sind so eindeutig, so vollkommmen, so hässlich... die schönen Bilder. Aber Wonnen eines triebhaften Balletts, die unwillkürliche, unausschöpfliche Natur selbst, das Chaos und urzeitliche Zeremonien lese ich aus Annoncenklischees, Reklamebildern, Briefmarken, Klebebogen, aus Kulissen für Kindertheater, Abziehbildern, Vignetten ; mich entzückt die Romantik des Geschmacklosen. »

35. Franz Kafka, La Métamorphose, in : Récits, Euvres complètes II, éd. Claude David, Paris, Gallimard (Pléiade), 1980, p. 192/Die Verwandlung, in : Ein Landarzt und andere Drucke zu Lebzeiten, kritische Ausgabe hrsg. Von W. Kittler, H.G. Koch und G. Neumann, Frankfurt/Main, Fischer, 1994, S. 93 : " Als Gregor Samsa eines Morgens aus unruhigen Träumen erwachte, fand er sich in seinem Bett zu einem ungeheuren Ungeziefer verwandelt. [...] "Was ist mit mir geschehen ?" dachte er. Es war kein Traum. "

36. F. Kafka, Description d'un combat (Ms A.), in : Récits, CEuvres complètes II, p. 20.

37. Zitiert in : Paul Vogt, Expressionismus. Deutsche Malerei zwischen 1905 und 1920, Köln, DuMont, 1978, S. 6 : « Niemals war der Mensch so klein. Niemals war ihm so bang. Da schreit die Not jetzt auf : der Mensch schreit nach seiner Seele, die ganze Zeit wird ein einziger Notschrei. Auch die Kunst schreit mit, in die tiefe Finsternis hinein, sie schreit um Hilfe, sie schreit nach Geist : das ist der Expressionismus. »

38. Carol S. Eliel (Hrsg.), Ludwig Meidner. Apokalyptische Landschaften. Berlinische Galerie, Museum für moderne Kunst, Martin-Gropius Bau, Berlin, Ausstellung vom 3.02 bis zum 8.04. 1990, München, Prestel, 1990, S. 71 : «Er ekelt sich vor sich selbst, er haßt sich selbst, er schneidet seinem Spiegelbild Fratzen und betrachtet, gleich einem Jäger auf dem Anstand, seine "gletscherblanke Glatze". Er macht auf sich selbst Jagd. Mit unbarmherziger, ingrimmiger Genauigkeit studiert er seine Physiognomie. Er findet Lust am Grimassieren, und unter dem Zwang solch unbarmherziger Lust der Selbstbeobachtung beginnt er zu malen, hohnlachend über 
sich selbst, ein gespenstisches Selbstporträt, dessen wildes Grinsen er der ganzen Welt entgegenstreckt. "

39. Walter Benjamin, Expérience et pauvreté in : Euvres II, trad. Par M. De Gandillac, R. Rochlitz et P. Rusch, Paris, Gallimard (Folio Essais), 2000, p.365/Erfahrung und Armut, in: Gesammelte Schriften, Bd. II, 1: Aufsätze, Essays, Vorträge, Frankfurt/Main, Suhrkamp, 1977, S. 214 : «Eine Generation, die noch mit der Pferdebahn zur Schule gefahren war, stand unter freiem Himmel in einer Landschaft, in der nichts unverändert geblieben war als die Wolken, und in der Mitte, in einem Kraftfeld zerstörender Ströme und Explosionen, der winzige gebrechliche Menschenkörper. »

40. Paul Vogt, Expressionismus. Deutsche Malerei zwischen 1905 und 1920, Köln, DuMOnt, 1978, S. 130 : "Jede Erscheinungsform der sichtbaren Wirklichkeit erscheint als Aufforderung zur geistigen Ordnung. Malerei befreit sich von den ästhetischen Normen, um zur moralischen Handlung zu werden. »

41. Matthias Eberle, Max Beckmann. Die Nacht. Passion ohne Erlösung, Frankfurt/Main, Fischer, 1984, S. 37, in : Beckmann, Catalogue de l'exposition du Centre Pompidou : «Beckmann, un peintre dans l'histoire », 10.9.2002-6.01. 2003, Paris, Éditions du Centre Pompidou, 2002, p. 76.

42. Cité in : Wolf-Dieter Dube, Les Expressionnistes, Paris, Thames and Hudson, 1996, p. 167.

43. Carl Einstein, Die Kunst des 20. Jahrhunderts, Berlin, Propyläen, 1926, S. 155.

44. Anz, S. 171-172 : « Das Widerliche und Ekelerregende in der Literatur des

Existenz fungiert nicht, [...] im Rahmen einer Totalisierung des Ästhetischen, die auch noch das Hässliche in sich integrieren will, sondern es ist hier Ausdruck einer veränderten Funktion von Literatur : sie sollte,... den erfahrenen Disharmonien und Widersprüchen nicht eine positive Gegenwelt entgegenhalten, sondern sie in ihren existentiellen Auswirkungen aufzeigen, und zwar in Opposition zu einer Gesellschaft, der daran gelegen sein musste, ihre Widersprüche ideologisch zu verschleiern. Literatur sollte menschliches Dasein nicht verschönern, angenehmer und erträglicher machen. Diese Aufgabe hatte bereits in pervertierter Form die herrschende Kulturindustrie übernommen. Sondern sie sollte beunruhigen, provozieren, eingefahrene Denkund Bewältigungsmuster radikal aufbrechen. »

45. Ibid., S. 175 : « Vor dem Hintergrund einer sich dynamisch wandelnden Gesellschaft erscheint die Negativität der Existenzliteratur in einem umgreifenden historischen Sinn höchst funktional, insofern sie in der Bewusstwerdung und Bewußtmachung des anachronistisch-desolaten Zustandes bestehender Denkformen den Anstoß zur Suche nach neuem gibt. Die Literatur der Existenz wird in der „Moderne” zu einer Instanz, die der Gesellschaft die andauernde Überprüfung ihrer Normen, Werte und Deutungsmuster abfordert. »

46. Adorno, Théorie esthétique, Paris, Klincksieck, 1989, p. 69-70/Ästhetische Theorie, Suhrkamp, Frankfurt, 2000, S. 74: «Daß Kunst im Begriff des Schönen nicht aufgeht sondern, um ihn zu erfüllen, des Hässlichen als seiner Negation bedurfte, ist ein Gemeinplatz ».

47. Ibid., p. 71/Ästhetische Theorie (A.T.), S. 76 : « Noch der vom bürgerlichen Bewusstsein naïv vollzogene Richtspruch über die Hässlichkeit der von Industrie zerwühlten Landschaft trifft eine Reaktion, die erscheinende Naturbeherrschung dort, wo Natur den Menschen die Fassade des Unbeherrschten zukehrt. Jede Entrüstung fügt darum der Ideologie von Herrschaft sich ein. Solche Hässlichkeit verschwände, wenn einmal das Verhältnis der Menschen zur Natur des repressiven Charakters sich entäußerte, der die Unterdrückung des Menschen fortsetzt, nicht umgekehrt. Das Potential dazu in der von Technik verwüsten Welt liegt in einer friedlich gewordenen Technik, nicht in eingeplanten Enklaven ».

48. Ibid, p. 73/Ästhetische Theorie, S. 78-79 : « Kunst muss das als hässlich Verfemte zu ihrer Sache machen, nicht länger um es zu integrieren, zu mildern oder durch den Humor, der abstoßender ist als alles Abstoßende, mit seiner Existenz zu versöhnen, sondern um im Hässlichen die Welt zu denunzieren, die es nach ihrem Bild schafft und reproduziert ».

49. M. Ribon, Esthétique de la catastrophe, Paris, Kimé, 1994, p. 215. 


\section{RÉSUMÉS}

Partant de l'éloge nietzschéen de l'oubli créateur et de sa justification de la laideur comme stimulant à la création artistique, cette contribution se propose de montrer comment l'oubli volontaire du beau au profit de l'exigence du laid provoque en Allemagne au début du xx siècle, dans la lignée de Hugo et Baudelaire, une remise en cause des canons esthétiques traditionnels soit de l'identité du Beau et du Bien : à l'« esthétique négative » développée par Nietzsche répond la «littérature existentielle» représentée ici par Rilke et Kafka, qui rompt avec le principe apollinien de stylisation du réel et dénonce la belle apparence comme duperie. Ce mouvement de déconstruction culmine dans l'art expressionniste de Meidner, Dix ou Beckmann, qui ouvre à l'artiste le champ du dionysiaque, du cri et du choc nécessaires à l'évolution de l'art vers une esthétique de l'expression et de la rébellion contre la laideur du monde et les effets négatifs du progrès technique.

Ausgehend von Nietzsches Lobpreisung des schöpferischen Vergessens und dessen Rechtfertigung des Hässlichen als Stimulans des künstlerischen Schaffens nimmt sich vorliegender Beitrag vor, zu zeigen, wie das willkürliche Vergessen des Schönen zugunsten der Forderung des Hässlichen im Deutschland des zwanzigsten Jahrhunderts im Anschluss an Hugo und Baudelaire eine Infragestellung der herkömmlichen ästhetischen Kanone - also der Identität des Schönen mit dem Guten - zur Folge hat: der sogenannten, von Nietzsche entwickelten « negativen Ästhetik » entspricht folglich die hier von Rilke und Kafka vertretene « Literatur der Existenz », welche mit dem apollinischen Prinzip der Stilisierung der Realität bricht und den schönen Schein als Täuschung entlarvt. Den Höhepunkt dieser dekonstruktiven Bewegung markiert folglich die expressionistische Kunst Meidners, Dix' und Beckmanns, welche dem Künstler den Bereich des Dionysischen, des Schreis und des Schocks als notwendige Bedingungen der Entwicklung der Kunst zur Ästhetik des Ausdrucks und der Auflehnung gegen die Hässlichkeit der Welt eröffnet und die negativen Folgen des technischen Fortschritts entpuppt.

\section{AUTEUR}

\section{FLORENCE BANCAUD}

Université de Rouen 